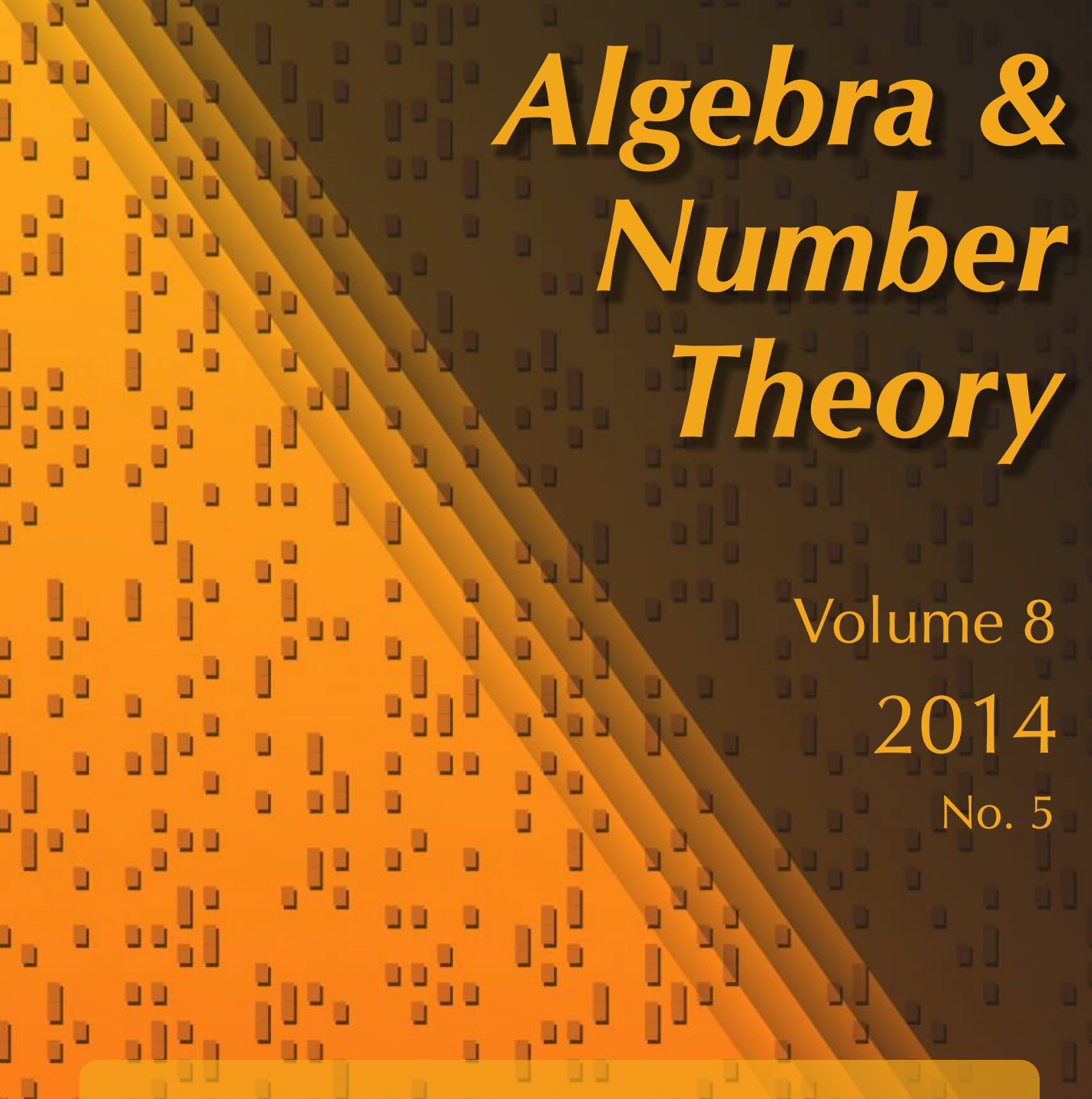

Poisson structures and star products on quasimodular forms

François Dumas and Emmanuel Royer

\lrcorner

\lrcorner$\lrcorner$
\lrcorner 


\title{
Poisson structures and star products on quasimodular forms
}

\author{
François Dumas and Emmanuel Royer
}

We construct and classify all Poisson structures on quasimodular forms that extend the one coming from the first Rankin-Cohen bracket on the modular forms. We use them to build formal deformations on the algebra of quasimodular forms.

\section{Introduction}

Henri Cohen [1975, Theorem 7.1] defined a collection of bidifferential operators on modular forms. Let $n$ be a positive integer, $f$ a modular form of weight $k$, and $g$ a modular form of weight $\ell$. The $n$-th Rankin-Cohen bracket of $f$ and $g$ is the modular form of weight $k+\ell+2 n$ defined by

$$
\mathrm{RC}_{n}(f, g)=\sum_{r=0}^{n}(-1)^{r}\left(\begin{array}{c}
k+n-1 \\
n-r
\end{array}\right)\left(\begin{array}{c}
\ell+n-1 \\
r
\end{array}\right) \mathrm{D}^{r} f \mathrm{D}^{n-r} g \quad\left(\mathrm{D}=\frac{1}{2 \pi \mathrm{i}} \frac{\mathrm{d}}{\mathrm{d} z}\right) .
$$

The algebraic structure of these brackets has been studied in the seminal [Zagier 1994]. That Rankin-Cohen brackets define a formal deformation of the algebra of modular forms has been widely studied. Important contributions are [Unterberger and Unterberger 1996; Cohen et al. 1997; Yao 2007; Bieliavsky et al. 2007; Pevzner 2012; Kobayashi and Pevzner 2013].

In this paper, we construct formal deformations of the algebra $\mu_{*}^{\leq \infty}$ of quasimodular forms. This algebra is generated over $\mathbb{C}$ by the three Eisenstein series $E_{2}, E_{4}$ and $E_{6}$. The algebra $M_{*}$ of modular forms is the subalgebra generated by $E_{4}$ and $E_{6}$. As a first step, we classify the admissible Poisson structures of $\mu_{*}^{\leq \infty}$. A Poisson bracket $\{$,$\} on \mu_{*}^{\leq \infty}$ is admissible if

(i) the restriction of $\{$,$\} to the algebra M_{*}$ of modular forms is the first RankinCohen bracket $\mathrm{RC}_{1}$ and

We thank François Martin and Anne Pichereau for many fruitful discussions. MSC2010: primary 17B63; secondary 11F25, 11F11, 16W25.

Keywords: quasimodular forms, Poisson brackets, Rankin-Cohen brackets, formal deformation,

Eholzer product, star product. 
(ii) it satisfies $\left\{\mathcal{M}_{k}^{\leq s}, \mathcal{M}_{\ell}^{\leq t}\right\} \subset \mathcal{M}_{k+\ell+2}^{\leq s+t}$ for any even integers $k, \ell$ and any integers $s$ and $t$,

where $M_{k}^{\leq s}$ is the vector space of quasimodular forms of weight $k$ and depth less than $s$. The vector space of parabolic modular forms of weight 12 is one-dimensional. We choose $\Delta=\mathrm{E}_{4}^{3}-\mathrm{E}_{6}^{2}$ a generator.

Proposition A (first family of Poisson brackets). For any $\lambda \in \mathbb{C}^{*}$, there exists an admissible Poisson bracket $\{,\}_{\lambda}$ on the algebra of quasimodular forms defined by the following values on the generators:

$$
\begin{aligned}
& \left\{\mathrm{E}_{4}, \mathrm{E}_{6}\right\}_{\lambda}=-2 \Delta, \\
& \left\{\mathrm{E}_{2}, \mathrm{E}_{4}\right\}_{\lambda}=-\frac{1}{3}\left(2 \mathrm{E}_{6} \mathrm{E}_{2}-\lambda \mathrm{E}_{4}^{2}\right), \\
& \left\{\mathrm{E}_{2}, \mathrm{E}_{6}\right\}_{\lambda}=-\frac{1}{2}\left(2 \mathrm{E}_{4}^{2} \mathrm{E}_{2}-\lambda \mathrm{E}_{4} \mathrm{E}_{6}\right) .
\end{aligned}
$$

Moreover:

(i) For any $\lambda \in \mathbb{C}^{*}$, the Poisson bracket $\{,\}_{\lambda}$ is not unimodular.

(ii) The Poisson algebras $\left(M_{*}^{\leq \infty},\{,\}_{\lambda}\right)$ and $\left(M_{*}^{\leq \infty},\{,\}_{\lambda^{\prime}}\right)$ are Poisson modular isomorphic for all $\lambda$ and $\lambda^{\prime}$ in $\mathbb{C}^{*}$.

(iii) For any $\lambda \in \mathbb{C}^{*}$, the Poisson centre of $\left(\mathcal{M}_{*}^{\leq \infty},\{,\}_{\lambda}\right)$ is $\mathbb{C}$.

Remark. A Poisson isomorphism $\varphi$ on $M_{*}^{\leq \infty}$ is modular if $\varphi\left(M_{*}\right) \subset M_{*}$.

Thanks to (ii) in Proposition A, we restrict to the bracket $\{,\}_{1}$. Following [Zagier 1994, Equation (38)], we consider the derivation w on $M_{*}^{\leq \infty}$ defined by

$$
\mathrm{w}(f)=\frac{\{\Delta, f\}_{1}}{12 \Delta} .
$$

A derivation $\delta$ on $M_{*}^{\leq \infty}$ is complex-like if $\delta\left(M_{k}^{\leq s}\right) \subset M_{k+2}^{\leq s+1}$ for any $k$ and $s$. The set of complex-like derivations $\delta$ such that $k f \delta(g)-\ell g \delta(f)=0$ for any $f \in M_{k}^{\leq s}$ and $g \in M_{\ell}^{\leq t}$, for any $k, \ell, s, t$, is a one-dimensional vector space over $\mathbb{C}$. Let $\pi$ be a generator. The following theorem provides a first family of formal deformations of the algebra $M_{*}^{\leq \infty}$.

Theorem B. For any $a \in \mathbb{C}$, let $\mathrm{d}_{a}$ be the derivation on $\mu_{*}^{\leq \infty}$ defined by $\mathrm{d}_{a}=a \pi+\mathrm{w}$.

(i) For all quasimodular forms $f$ and $g$ of respective weights $k$ and $\ell$, we have

$$
\{f, g\}_{1}=k f \mathrm{~d}_{a}(g)-\ell g \mathrm{~d}_{a}(f) .
$$

(ii) More generally, for any $a \in \mathbb{C}$, the brackets defined for any integer $n \geq 0$ by

$[f, g]_{\mathrm{d}_{a}, n}=\sum_{r=0}^{n}(-1)^{r}\left(\begin{array}{c}k+n-1 \\ n-r\end{array}\right)\left(\begin{array}{c}\ell+n-1 \\ r\end{array}\right) \mathrm{d}_{a}^{r}(f) \mathrm{d}_{a}^{n-r}(g)$

$$
\left(f \in M_{k}^{\leq \infty}, g \in M_{\ell}^{\leq \infty}\right)
$$


satisfy

$$
\left[\mu_{k}^{\leq \infty}, \mu_{\ell}^{\leq \infty}\right]_{\mathrm{d}_{a}, n} \subset \mathcal{M}_{k+\ell+2 n}^{\leq \infty}
$$

and define a formal deformation of $\mu_{*}^{\leq \infty}$.

(iii) Moreover, $\left[\mathcal{M}_{k}^{\leq s}, \mathcal{M}_{\ell}^{\leq t}\right]_{\mathrm{d}_{a}, n} \subset \mathcal{M}_{k+\ell+2 n}^{\leq s+t}$ for all $n, s, t, k, \ell$ if and only if $a=0$.

Remark. A generator $\pi$ is defined by linear extension of $\pi(f)=k f \mathrm{E}_{2}$ for $f$ any quasimodular form of weight $k$. For this choice, the derivation $\mathrm{d}_{a}$ is defined on the generators by

$$
\mathrm{d}_{a} \mathrm{E}_{2}=2 a \mathrm{E}_{2}^{2}-\frac{1}{12} \mathrm{E}_{4}, \quad \mathrm{~d}_{a} \mathrm{E}_{4}=4 a \mathrm{E}_{4} \mathrm{E}_{2}-\frac{1}{3} \mathrm{E}_{6}, \quad \mathrm{~d}_{a} \mathrm{E}_{6}=6 a \mathrm{E}_{6} \mathrm{E}_{2}-\frac{1}{2} \mathrm{E}_{4}^{2} .
$$

Proposition $\mathbf{C}$ (second family of Poisson brackets). For any $\alpha \in \mathbb{C}$, there exists an admissible Poisson bracket (, $)_{\alpha}$ on the algebra of quasimodular forms defined by the following values on the generators:

$$
\left(\mathrm{E}_{4}, \mathrm{E}_{6}\right)_{\alpha}=-2 \Delta, \quad\left(\mathrm{E}_{2}, \mathrm{E}_{4}\right)_{\alpha}=\alpha \mathrm{E}_{6} \mathrm{E}_{2}, \quad\left(\mathrm{E}_{2}, \mathrm{E}_{6}\right)_{\alpha}=\frac{3}{2} \alpha \mathrm{E}_{4}^{2} \mathrm{E}_{2} .
$$

Moreover:

(i) For any $\alpha \in \mathbb{C} \backslash\{4\}$, the Poisson bracket (, , $)_{\alpha}$ is not unimodular. For $\alpha=4$, the Poisson bracket $(,)_{4}$ is Jacobian (and hence unimodular) of potential $k_{0}=-2 \Delta \mathrm{E}_{2}$.

(ii) The Poisson algebras $\left(\mu_{*}^{\leq \infty},(,)_{\alpha}\right)$ and $\left(\mu_{*}^{\leq \infty},(,)_{\alpha^{\prime}}\right)$ are Poisson modular isomorphic if and only if $\alpha=\alpha^{\prime}$.

(iii) For any $\alpha \in \mathbb{C}$,

(a) if $\alpha \notin \mathbb{Q}$, the Poisson centre of $\left(\mu_{*}^{\leq \infty},(,)_{\alpha}\right)$ is $\mathbb{C}$;

(b) if $\alpha=0$, the Poisson centre of $\left(\mu_{*}^{\leq \infty},(,)_{\alpha}\right)$ is $\mathbb{C}\left[\mathrm{E}_{2}\right]$;

(c) if $\alpha=p / q$ with $p \in \mathbb{Z}^{*}$ and $q \in \mathbb{N}^{*}, p$ and $q$ coprimes, the Poisson centre of $\left(\mathcal{M}_{*}^{\leq \infty},(,)_{\alpha}\right)$ is

$$
\begin{cases}\mathbb{C} & \text { if } p<0 \\ \mathbb{C}\left[\Delta^{p} \mathrm{E}_{2}^{4 q}\right] & \text { if } p \geq 1 \text { is odd } \\ \mathbb{C}\left[\Delta^{u} \mathrm{E}_{2}^{2 q}\right] & \text { if } p=2 u \text { for odd } u \geq 1 \\ \mathbb{C}\left[\Delta^{v} \mathrm{E}_{2}^{q}\right] & \text { if } p=4 v \text { with } v \geq 1\end{cases}
$$

Remark. The bracket $(,)_{0}$ is the trivial bracket.

This second family provides a new set of formal deformations of the algebra of quasimodular forms. Following [Zagier 1994, Equation (38)], we consider the derivation $\mathrm{v}$ defined on $\mu_{*}^{\leq \infty}$ by

$$
\mathrm{v}(f)=\frac{(\Delta, f)_{\alpha}}{12 \Delta} .
$$


Let us define $\mathscr{K}_{\alpha}: \mathcal{M}_{*}^{\leq \infty} \rightarrow \mathbb{C}$ by setting $\mathcal{K}_{\alpha}(f)=k-(3 \alpha+2) s$ if $f$ has weight $k$ and depth $s$. The set of complex-like derivations $\delta$ such that

$$
\mathscr{K}_{\alpha}(f) f \delta(g)-\mathscr{K}_{\alpha}(g) g \delta(f)=0
$$

for any $f \in \mathcal{M}_{k}^{\leq s}$ and $g \in \mathcal{M}_{\ell}^{\leq t}$, for any $k, \ell, s, t$, is a one-dimensional vector space over $\mathbb{C}$. Let $\pi_{\alpha}$ be a generator. We define

$$
M_{k}^{s}=M_{k-2 s} \mathrm{E}_{2}^{s} .
$$

Theorem D. Let $\alpha \in \mathbb{C}$. For any $b \in \mathbb{C}$, let $\delta_{\alpha, b}$ be the derivation on $\mu_{*}^{\leq \infty}$ defined by $\delta_{\alpha, b}=b \pi_{\alpha}+\mathrm{v}$.

(i) For all $f \in \mathcal{M}_{k}^{s}$ and $g \in \mathcal{M}_{\ell}^{t}$, we have

$$
(f, g)_{\alpha}=(k-(3 \alpha+2) s) f \delta_{\alpha, b}(g)-(\ell-(3 \alpha+2) t) g \delta_{\alpha, b}(f)
$$

for any $f \in \mathcal{M}_{k}^{s}$ and $g \in \mathcal{M}_{\ell}^{t}$.

(ii) Moreover, the brackets defined for any integer $n \geq 0$ by

$[f, g]_{\delta_{\alpha, b}, n}^{\mathscr{K}_{\alpha}}$

$$
=\sum_{r=0}^{n}(-1)^{r}\left(\begin{array}{c}
k-(3 \alpha+2) s+n-1 \\
n-r
\end{array}\right)\left(\begin{array}{c}
\ell-(3 \alpha+2) t+n-1 \\
r
\end{array}\right) \delta_{\alpha, b}^{r}(f) \delta_{\alpha, b}^{n-r}(g)
$$

for any $f \in \mathcal{M}_{k}^{s}$ and $g \in \mathcal{M}_{\ell}^{t}$ define a formal deformation of $\mathcal{M}_{*}^{\leq \infty}$ satisfying

$$
\left[\mathcal{M}_{k}^{\leq s}, \mathcal{M}_{\ell}^{\leq t}\right]_{\delta_{\alpha, b}, n}^{\mathscr{K}_{\alpha}} \subset \mathcal{M}_{k+\ell+2 n}^{\leq s+t}
$$

for all $k, \ell$ in $2 \mathbb{N}$ and $s, t$ in $\mathbb{N}$ if and only if $b=0$.

Remark. A generator $\pi_{\alpha}$ is defined by linear extension of

$$
\pi_{\alpha}(f)=[k-(3 \alpha+2) s] f \mathrm{E}_{2} \quad\left(f \in \mathcal{M}_{k}^{s}\right) .
$$

For this choice, the derivation $\delta_{\alpha, b}$ is defined on the generators by

$$
\delta_{\alpha, b} \mathrm{E}_{2}=-3 b \alpha \mathrm{E}_{2}^{2}, \quad \delta_{\alpha, b} \mathrm{E}_{4}=4 b \mathrm{E}_{4} \mathrm{E}_{2}-\frac{1}{3} \mathrm{E}_{6}, \quad \delta_{\alpha, b} \mathrm{E}_{6}=6 b \mathrm{E}_{6} \mathrm{E}_{2}-\frac{1}{2} \mathrm{E}_{4}^{2} .
$$

To complete the classification of Poisson structures, we introduce a third family of Poisson brackets. We note, however, that when $\mu \neq 0$ this third family does not lead to a formal deformation of $\mu_{*}^{\leq \infty}$ with the shape of Rankin-Cohen brackets (see Section 4.3).

Proposition $\mathbf{E}$ (third family of Poisson brackets). For any $\mu \in \mathbb{C}$, there exists an admissible Poisson bracket $\langle,\rangle_{\mu}$ on the algebra of quasimodular forms defined by 
the following values on the generators:

$$
\begin{aligned}
& \left\langle\mathrm{E}_{4}, \mathrm{E}_{6}\right\rangle_{\mu}=-2 \Delta, \\
& \left\langle\mathrm{E}_{2}, \mathrm{E}_{4}\right\rangle_{\mu}=4 \mathrm{E}_{6} \mathrm{E}_{2}+\mu \mathrm{E}_{4}^{2}, \\
& \left\langle\mathrm{E}_{2}, \mathrm{E}_{6}\right\rangle_{\mu}=6 \mathrm{E}_{4}^{2} \mathrm{E}_{2}-2 \mu \mathrm{E}_{4} \mathrm{E}_{6} .
\end{aligned}
$$

Moreover:

(i) This Poisson bracket is Jacobian with potential

$$
k_{\mu}=-2 \Delta \mathrm{E}_{2}+\mu \mathrm{E}_{4}^{2} \mathrm{E}_{6} .
$$

(ii) The Poisson algebras $\left(\mu_{*}^{\leq \infty},\langle,\rangle_{\mu}\right)$ and $\left(\mu_{*}^{\leq \infty},\langle,\rangle_{\mu^{\prime}}\right)$ are Poisson modular isomorphic for all $\mu$ and $\mu^{\prime}$ in $\mathbb{C}^{*}$.

(iii) For any $\mu \in \mathbb{C}$, the Poisson centre of $\left(\mu_{*}^{\leq \infty},\langle,\rangle_{\mu}\right)$ is the polynomial algebra $\mathbb{C}\left[k_{\mu}\right]$.

Remark. We note that $\langle,\rangle_{0}=(,)_{4}$.

Finally, the following result implies that our classification is complete.

Theorem F. Up to Poisson modular isomorphism, the only distinct admissible Poisson brackets on the algebra of quasimodular forms are $\{,\}_{1},\langle,\rangle_{1}$ and the family $(,)_{\alpha}$ for any $\alpha \in \mathbb{C}$.

Remark. We could endow the algebra of modular forms with another Poisson structure $\mathrm{b}$. If we require $\mathrm{b}\left(M_{k}, M_{\ell}\right) \subset M_{k+\ell+2}$, then $\mathrm{b}$ is necessarily defined by $\mathrm{b}\left(\mathrm{E}_{4}, \mathrm{E}_{6}\right)=\alpha \mathrm{E}_{4}^{3}+\beta \mathrm{E}_{6}^{2}$ for some complex numbers $\alpha$ and $\beta$. If $\alpha \beta \neq 0$, then $\left(\mu_{*}, \mathrm{~b}\right)$ is Poisson isomorphic to $\left(M_{*}, \mathrm{RC}_{1}\right)$ and is indeed studied by this work. If $\alpha \beta=0$, the Poisson algebras are no longer Poisson isomorphic (they do not have the same group of automorphisms). This degenerate case deserves another study.

Remark. From an algebraic point of view, classifications of Poisson structures and associated (co)homology for polynomial algebras in two variables appear in [Monnier 2002; Pichereau 2006a; Roger and Vanhaecke 2002] for a Poisson bracket on $\mathbb{C}[x, y]$ defined by $\{x, y\}=\varphi(x, y)$ with $\varphi$ a homogeneous or square-free weighthomogeneous polynomial in $\mathbb{C}[x, y]$. The algebra of modular forms $M_{*}=\mathbb{C}\left[\mathrm{E}_{4}, \mathrm{E}_{6}\right]$ with the Poisson bracket defined by $\mathrm{RC}_{1}$ is the case $A_{2}$ in the classification theorem 3.8 in [Monnier 2002]. Applying Propositions 4.10 and 4.11 of [Pichereau 2006a], or Theorems 4.6 and 4.11 of [Monnier 2002], we can deduce that the Poisson cohomology spaces $\operatorname{HP}^{1}\left(M_{*}\right)$ and $\operatorname{HP}^{2}\left(M_{*}\right)$ are of respective dimensions 1 and 2 . In three variables, the Poisson structures on the algebra $\mu_{*}^{\leq \infty}=\mathbb{C}\left[\mathrm{E}_{2}, \mathrm{E}_{4}, \mathrm{E}_{6}\right]$ of quasimodular forms arising from Theorem $\mathrm{F}$ do not fall under the classification of [Dufour and Haraki 1991] since they are not quadratic. The (co)homological study of Pichereau [2006a; 2006b] does not apply to the brackets $\{,\}_{1}$ and $(,)_{\alpha}$, since 
they are not Jacobian, or to the Jacobian bracket $\langle,\rangle_{1}$, because its potential $k_{1}$ does not admit an isolated singularity at the origin.

\section{Number theoretic and algebraic background}

2.1. Quasimodular forms. The aim of this section is to provide the necessary background on quasimodular forms. For details, the reader is advised to refer to [Zagier 2008] or [Martin and Royer 2005]. On SL(2, Z ), a modular form of weight $k \in 2 \mathbb{N}, k \neq 2$, is a holomorphic function on the Poincaré upper half-plane $\mathscr{H}=\{z \in \mathbb{C}: \mathfrak{I} z>0\}$ satisfying

$$
(c z+d)^{-k} f\left(\frac{a z+b}{c z+d}\right)=f(z)
$$

for any $\left(\begin{array}{ll}a & b \\ c & d\end{array}\right) \in \operatorname{SL}(2, \mathbb{Z})$ and having Fourier expansion

$$
f(z)=\sum_{n \geq 0} \widehat{f}(n) \mathrm{e}^{2 \pi \mathrm{i} n z} .
$$

We denote by $M_{k}$ the finite-dimensional space of modular forms of weight $k$. The algebra of modular forms is defined as the graded algebra

$$
\mu_{*}=\bigoplus_{\substack{k \in 2 \mathbb{N} \\ k \neq 2}} \mu_{k}
$$

Let $k \geq 2$ be even. We define the Eisenstein series of weight $k$ by

$$
\mathrm{E}_{k}(z)=1-\frac{2 k}{B_{k}} \sum_{n=1}^{+\infty} \sigma_{k-1}(n) \mathrm{e}^{2 \pi \mathrm{i} n z} .
$$

Here the rational numbers $B_{k}$ are defined by their exponential generating series

$$
\sum_{n=0}^{+\infty} B_{n} \frac{t^{n}}{n !}=\frac{t}{\mathrm{e}^{t}-1}
$$

and $\sigma_{k-1}$ is the divisor function defined by

$$
\sigma_{k-1}(n)=\sum_{\substack{d \mid n \\ d>0}} d^{k-1} \quad\left(n \in \mathbb{N}^{*}\right) .
$$

If $k \geq 4$, the Eisenstein series $\mathrm{E}_{k}$ is a modular form of weight $k$ and $\mu_{*}$ is the polynomial algebra in the two algebraically independent Eisenstein series $\mathrm{E}_{4}$ and $\mathrm{E}_{6}$. In other words,

$$
M_{*}=\mathbb{C}\left[\mathrm{E}_{4}, \mathrm{E}_{6}\right], \quad M_{k}=\bigoplus_{\substack{(i, j) \in \mathbb{N}^{2} \\ 4 i+6 j=k}} \mathbb{C E}_{4}^{i} \mathrm{E}_{6}^{j}
$$


However, the Eisenstein series $\mathrm{E}_{2}$ is not a modular form. It satisfies

$$
(c z+d)^{-2} \mathrm{E}_{2}\left(\frac{a z+b}{c z+d}\right)=\mathrm{E}_{2}(z)+\frac{6}{\pi \mathrm{i}} \frac{c}{c z+d} \quad(z \in \mathscr{H})
$$

for any $\left(\begin{array}{ll}a & b \\ c & d\end{array}\right) \in \operatorname{SL}(2, \mathbb{Z})$. Moreover, the algebra of modular forms is not stable by the normalised complex derivation

$$
\mathrm{D}=\frac{1}{2 \pi \mathrm{i}} \frac{\mathrm{d}}{\mathrm{d} z} .
$$

For example, we have the Ramanujan differential equations

$$
\mathrm{DE}_{2}=\frac{1}{12}\left(\mathrm{E}_{2}^{2}-\mathrm{E}_{4}\right), \quad \mathrm{DE}_{4}=\frac{1}{3}\left(\mathrm{E}_{4} \mathrm{E}_{2}-\mathrm{E}_{6}\right), \quad \mathrm{DE}_{6}=\frac{1}{2}\left(\mathrm{E}_{6} \mathrm{E}_{2}-\mathrm{E}_{4}^{2}\right) .
$$

To account for these observations, and using the fact that $\mathrm{E}_{2}, \mathrm{E}_{4}$ and $\mathrm{E}_{6}$ are algebraically independent, we introduce the algebra $\mu_{*}^{\leq \infty}$ of quasimodular forms defined as the polynomial algebra

$$
\mu_{*}^{\leq \infty}=\mathbb{C}\left[\mathrm{E}_{2}, \mathrm{E}_{4}, \mathrm{E}_{6}\right]=\mu_{*}\left[\mathrm{E}_{2}\right] .
$$

More intrinsically, if for $\gamma=\left(\begin{array}{ll}a & b \\ c & d\end{array}\right) \in \operatorname{SL}(2, \mathbb{Z})$ we define

and

$$
\mathrm{X}(\gamma)=z \mapsto \frac{c}{c z+d}
$$

$$
\left.f\right|_{k} \gamma=z \mapsto(c z+d)^{-k} f\left(\frac{a z+b}{c z+d}\right),
$$

then a quasimodular form of weight $k \in 2 \mathbb{N}$ and depth $s \in \mathbb{N}$ is a holomorphic function $f$ on $\mathscr{H}$ such that there exist holomorphic functions $f_{0}, \ldots, f_{s}\left(f_{s} \neq 0\right)$ satisfying

$$
\left.f\right|_{k} \gamma=\sum_{j=0}^{s} f_{j} \mathrm{X}(\gamma)^{j}
$$

for any $\gamma \in \operatorname{SL}(2, \mathbb{Z})$. Moreover, it is required that any $f_{j}$ have a Fourier expansion

$$
f_{j}(z)=\sum_{n \geq 0} \widehat{f}_{j}(n) \mathrm{e}^{2 \pi \mathrm{i} n z} \quad(z \in \mathscr{H}) .
$$

The zero function is supposed to have arbitrary weight and depth 0 . We write $\mu_{k}^{\leq \infty}$ for the space of quasimodular forms of weight $k$ and $\mu_{k}^{\leq s}$ for the space of quasimodular forms of weight $k$ and depth less than or equal to $s$. We have $\mu_{k}^{\leq 0}=\mu_{k}$ and

$$
\mu_{k}^{\leq s}=\bigoplus_{j=0}^{s} \mu_{k-2 j} \mathrm{E}_{2}^{j}, \quad \mu_{*}^{\leq \infty}=\bigoplus_{k \in 2 \mathbb{N}} \mu_{k}^{\leq k / 2}
$$


Moreover, D $\mu_{k}^{\leq s} \subset \mathcal{M}_{k+2}^{\leq s+1}$. Since the depth of a quasimodular form is nothing but its degree as a polynomial in $\mathrm{E}_{2}$ with modular coefficients, we note that

where

$$
\mu_{k}^{\leq s}=\bigoplus_{t=0}^{s} \mu_{k}^{t}, \quad \mu_{*}^{\leq \infty}=\bigoplus_{k \in 2 \mathbb{N}} \bigoplus_{t=0}^{k / 2} \mu_{k}^{t},
$$

$$
M_{k}^{t}=M_{k-2 t} \mathrm{E}_{2}^{t}=\bigoplus_{\substack{(i, j) \in \mathbb{N}^{2} \\ 4 i+6 j=k-2 t}} \mathbb{C E}_{4}^{i} \mathrm{E}_{6}^{j} \mathrm{E}_{2}^{t} .
$$

An important element in our study will be the discriminant function $\Delta=\mathrm{E}_{4}^{3}-\mathrm{E}_{6}^{2}$. We note that $\mathrm{D} \Delta=\Delta \mathrm{E}_{2}$.

Let $n$ be a nonnegative integer, $f$ a modular form of weight $k$, and $g$ a modular form of weight $\ell$. The $n$-th Rankin-Cohen bracket of $f$ and $g$ is

$$
\mathrm{RC}_{n}(f, g)=\sum_{r=0}^{n}(-1)^{r}\left(\begin{array}{c}
k+n-1 \\
n-r
\end{array}\right)\left(\begin{array}{c}
\ell+n-1 \\
r
\end{array}\right) \mathrm{D}^{r} f \mathrm{D}^{n-r} g .
$$

This is a modular form of weight $k+\ell+2 n$. If $f$ and $g$ are quasimodular forms of respective weights $k$ and $\ell$ and respective depths $s$ and $t$, their $n$-th Rankin-Cohen bracket is defined in [Martin and Royer 2009] by

$$
\mathrm{RC}_{n}(f, g)=\sum_{r=0}^{n}(-1)^{r}\left(\begin{array}{c}
k-s+n-1 \\
n-r
\end{array}\right)\left(\begin{array}{c}
\ell-t+n-1 \\
r
\end{array}\right) \mathrm{D}^{r} f \mathrm{D}^{n-r} g .
$$

This is a quasimodular form of weight $k+\ell+2 n$ and minimal depth (that is $s+t$ ).

2.2. Poisson algebra. The aim of this section is to give a brief account of what is needed about Poisson algebra. For more details, the reader is advised to refer to [Laurent-Gengoux et al. 2013]. A commutative $\mathbb{C}$-algebra $A$ is a Poisson algebra if there exists a bilinear skew-symmetric map $\mathrm{b}: A \times A \rightarrow A$ satisfying the two conditions

- (Leibniz rule) $\mathrm{b}(f g, h)=f \mathrm{~b}(g, h)+\mathrm{b}(f, h) g$ and

- (Jacobi identity) $\mathrm{b}(f, \mathrm{~b}(g, h))+\mathrm{b}(g, \mathrm{~b}(h, f))+\mathrm{b}(h, \mathrm{~b}(f, g))=0$

for all $f, g$ and $h$ in $A$. The bilinear map b is given the name of Poisson bracket. If $A$ is a finitely generated algebra with generators $x_{1}, \ldots, x_{N}$, a Poisson bracket $\mathrm{b}$ is entirely determined by its values $\mathrm{b}\left(x_{i}, x_{j}\right)$ for $i<j$, where $A$ is generated by $x_{1}, \ldots, x_{N}$. More precisely, we have

$$
\mathrm{b}(f, g)=\sum_{0 \leq i<j \leq N}\left(\frac{\partial f}{\partial x_{i}} \frac{\partial g}{\partial x_{j}}-\frac{\partial g}{\partial x_{i}} \frac{\partial f}{\partial x_{j}}\right) \mathrm{b}\left(x_{i}, x_{j}\right)
$$

for $f$ and $g$ expressed as polynomials in $x_{1}, \ldots, x_{N}$. 
If $A=\mathbb{C}[x, y]$, any $p \in A$ determines a Poisson bracket satisfying $\mathrm{b}(x, y)=p$. However, if $A=\mathbb{C}[x, y, z]$, for any $p, q$ and $r$ in $A$, there exists a Poisson bracket on $A$ defined by

$$
\mathrm{b}(x, y)=r, \quad \mathrm{~b}(y, z)=p \quad \text { and } \quad \mathrm{b}(z, x)=q
$$

if and only if

$$
\operatorname{curl}(p, q, r) \cdot(p, q, r)=0
$$

where

$$
\operatorname{curl}(p, q, r)=\left(\frac{\partial r}{\partial y}-\frac{\partial q}{\partial z}, \frac{\partial p}{\partial z}-\frac{\partial r}{\partial x}, \frac{\partial q}{\partial x}-\frac{\partial p}{\partial y}\right) .
$$

If condition (3) is satisfied, then $(p, q, r)$ is called a Poissonian triple. A particular case is obtained if there exists $k \in \mathbb{C}[x, y, z]$ such that $\operatorname{curl}(p, q, r)=$ $(p, q, r) \wedge \operatorname{grad} k$. The bracket $\mathrm{b}$ is said then to be unimodular. Among unimodular brackets are the Jacobian brackets. A bracket $\mathrm{b}$ is Jacobian if $(p, q, r)=\operatorname{grad} k$ for some polynomial $k$. The bracket $\mathrm{b}$ then satisfies

$$
\mathrm{b}(f, g)=\operatorname{jac}(f, g, k) \quad(f, g \in A) .
$$

In this case, $\mathbb{C}[x, y, z]$ is said to have a Jacobian Poisson structure of potential (or Casimir function) $k$. The Poissonian triple $(p, q, r)$ is said then to be exact.

The Poisson centre (or zeroth Poisson cohomology group) of a Poisson algebra $A$ is the Poisson subalgebra

$$
\operatorname{HP}^{0}(A)=\{g \in A: \mathrm{b}(f, g)=0, \forall f \in A\} .
$$

The Poisson centre is contained in the Poisson centraliser of any element in the algebra: let $f \in A$; its Poisson centraliser is $\{g \in A: \mathrm{b}(f, g)=0\}$. The following lemma computes the Poisson centre of polynomial algebras in three variables equipped with a Jacobian Poisson structure. It allows one to recover, for example, Proposition 4.2 of [Pichereau 2006b] in the particular case where the potential is a weight-homogeneous polynomial with an isolated singularity. A polynomial $h \in \mathbb{C}[x, y, z]$ is indecomposable if there is no polynomial $p \in \mathbb{C}[x]$ with $\operatorname{deg} p \geq 2$ such that $h=p \circ \ell$ for some $\ell \in \mathbb{C}[x, y, z]$.

Lemma 1. Let $\mathbb{C}[x, y, z]$ be endowed with a Jacobian Poisson structure of nonconstant potential $k$. Its Poisson centre is $\mathbb{C}[k]$ if and only if $k$ is indecomposable.

Proof. Assume that $k$ is not indecomposable: $k=p \circ \ell$ with $p \in \mathbb{C}[x], \operatorname{deg} p=2$. Then $\operatorname{jac}(\ell, g, k)=\left(p^{\prime} \circ \ell\right) \operatorname{jac}(\ell, g, \ell)$, and hence $\ell$ is in the Poisson centre, but not in $\mathbb{C}[k]$. Assume conversely that $k$ is indecomposable. Let $f$ be in the Poisson centre; then the rank of the Jacobian matrix of $(f, g, k)$ is at most 2 for any $g$. If it is 1 for any $g$ then $\operatorname{grad} f$ and $\operatorname{grad} k$ are zero, which contradicts the fact that $k$ is not constant. Hence, for some $g$, the rank is 2. It follows (see, for example, 
[Gutierrez and Sevilla 2006, Theorem 6]) that there exist $q \in \mathbb{C}[x, y, z], F \in \mathbb{C}[x]$ and $K \in \mathbb{C}[x]$ such that $f=F \circ q$ and $k=K \circ q$. Since $k$ is indecomposable and nonconstant, we have $\operatorname{deg} K=1$, and hence $q$ and $f$ are polynomials in $k$.

If $A$ and $B$ are two Poisson algebras with respective Poisson brackets $\mathrm{b}_{A}$ and $\mathrm{b}_{B}$, a map $\varphi: A \rightarrow B$ is a morphism of Poisson algebras when it is a morphism of algebras that satisfies

$$
\varphi\left(\mathrm{b}_{A}(f, g)\right)=\mathrm{b}_{B}(\varphi(f), \varphi(g))
$$

for any $f$ and $g$ in $A$. Two Poisson-isomorphic Poisson algebras have isomorphic Poisson centres.

We detail now a canonical way to extend a Poisson structure from an algebra $A$ to a polynomial algebra $A[x]$. This construction is due to Sei-Qwon Oh [2006]. A Poisson derivation of $A$ is a derivation $\sigma$ of $A$ satisfying

$$
\sigma(\mathrm{b}(f, g))=\mathrm{b}(\sigma(f), g)+\mathrm{b}(f, \sigma(g))
$$

for all $f$ and $g$ in $A$. If $\sigma$ is a Poisson derivation of $A$, a Poisson $\sigma$-derivation is a derivation $\delta$ of $A$ such that

$$
\delta(\mathrm{b}(f, g))=\mathrm{b}(\delta(f), g)+\mathrm{b}(f, \delta(g))+\sigma(f) \delta(g)-\delta(f) \sigma(g)
$$

for all $f$ and $g$ in $A$.

Theorem 2 [Oh 2006]. Let $\left(A, \mathrm{~b}_{A}\right)$ be a Poisson algebra. Let $\sigma$ and $\delta$ be linear maps on $A$. The polynomial ring $A[x]$ becomes a Poisson algebra with Poisson brackets $\mathrm{b}$ defined by

$$
\mathrm{b}(f, g)=\mathrm{b}_{A}(f, g), \quad \mathrm{b}(x, f)=\sigma(f) x+\delta(f)
$$

for all $f$ and $g$ in $A$ if and only if $\sigma$ is a Poisson derivation and $\delta$ is a Poisson $\sigma$-derivation. In this case, the Poisson algebra $A[x]$ is said to be a Poisson-Ore extension of $A$. It is denoted by $A[x]_{\sigma, \delta}$.

We describe also a general process to obtain Poisson brackets from a pair of derivations. A pair $(\delta, \mathrm{d})$ of two derivations of $A$ is solvable if there exists some scalar $\alpha$ such that $\delta \circ \mathrm{d}-\mathrm{d} \circ \delta=\alpha \mathrm{d}$. In particular, a solvable pair $(\delta, \mathrm{d})$ is abelian when $\alpha=0$.

Proposition 3. Let $A$ be a commutative algebra, and $\mathrm{d}$ and $\delta$ two derivations of $A$. Let $\mathrm{b}: A \times A \rightarrow A$ be defined by

$$
\mathrm{b}(f, g)=\delta(f) \mathrm{d}(g)-\mathrm{d}(f) \delta(g) \quad(f, g \in A) .
$$

Then:

(i) The map b is bilinear skew-symmetric and satisfies the Leibniz rule. 
(ii) If $(\delta, \mathrm{d})$ is solvable, then $\mathrm{b}$ satisfies the Jacobi identity and so becomes a Poisson bracket.

(iii) If $(\delta, \mathrm{d})$ is solvable, then $\mathrm{d}$ is a Poisson derivation for $\mathrm{b}$.

Proof. Point (i) is immediate. Point (ii) is a consequence of the following computation. If $(\delta, \mathrm{d})$ is solvable with $\delta \mathrm{d}-\mathrm{d} \delta=\alpha \mathrm{d}$ and if $\mathrm{B}: A \otimes A \otimes A \rightarrow A$ is defined by $\mathrm{B}(f, g, h)=\mathrm{b}(f, \mathrm{~b}(g, h))$, then

$\mathrm{B}=\alpha(\mathrm{d} \otimes \mathrm{d} \otimes \delta-\mathrm{d} \otimes \delta \otimes \mathrm{d})+(\delta \otimes(\mathrm{d} \circ \delta) \otimes \mathrm{d}-\mathrm{d} \otimes \delta \otimes(\mathrm{d} \circ \delta))$

$+(\mathrm{d} \otimes(\mathrm{d} \circ \delta) \otimes \delta-\delta \otimes \mathrm{d} \otimes(\mathrm{d} \circ \delta))+\left(\delta \otimes \delta \otimes \mathrm{d}^{2}-\delta \otimes \mathrm{d}^{2} \otimes \delta\right)+\left(\mathrm{d} \otimes \mathrm{d} \otimes \delta^{2}-\mathrm{d} \otimes \delta^{2} \otimes \mathrm{d}\right)$.

Point (iii) is obtained by direct computation.

A direct consequence of this proposition is the following corollary. If $A=$ $\bigoplus_{n \geq 0} A_{n}$ is a commutative graded algebra, a map $\kappa: A \rightarrow \mathbb{C}$ is graded-additive if for any $f \in A_{k}$ and $g \in A_{\ell}$ (for any $k$ and $\ell$ ) we have $\kappa(f g)=\kappa(f)+\kappa(g)$.

Corollary 4. Let $A=\bigoplus_{n \geq 0} A_{n}$ be a commutative graded algebra. Let $\kappa: A \rightarrow \mathbb{C}$ be a graded-additive map. Let $\mathrm{d}$ be a homogeneous derivation of $A$ (there exists $e \geq 0$ such that $\mathrm{d} A_{n} \subset A_{n+e}$ for any $e \geq 0$ ). Then the bracket defined on $A$ by the bilinear extension of

$$
\mathrm{b}(f, g)=\kappa(f) f \mathrm{~d}(g)-\kappa(g) g \mathrm{~d}(f) \quad\left(f \in A_{k}, g \in A_{\ell}\right)
$$

is a Poisson bracket for which $\mathrm{d}$ is a Poisson derivation.

We turn to formal deformations of a commutative $\mathbb{C}$-algebra $A$. Assume we have a family $\mu=\left(\mu_{i}\right)_{i \in \mathbb{N}}$ of bilinear maps $\mu_{i}: A \times A \rightarrow A$ such that $\mu_{0}$ is the product. Let $A \llbracket \hbar \rrbracket$ be the commutative algebra of formal power series in one variable $\hbar$ with coefficients in $A$. The family $\mu$ is a formal deformation of $A$ if the noncommutative product on $A \llbracket \hbar \rrbracket$ defined by extension of

$$
f * g=\sum_{j \geq 0} \mu_{j}(f, g) \hbar^{j} \quad(f, g \in A)
$$

is associative. This condition is equivalent to

$$
\sum_{r=0}^{n} \mu_{n-r}\left(\mu_{r}(f, g), h\right)=\sum_{r=0}^{n} \mu_{n-r}\left(f, \mu_{r}(g, h)\right) \quad(\text { for all } f, g, h \in A)
$$

for all $n \geq 0$. In this case, the product $*$ is called a star product. If $\mu$ is a formal deformation and if moreover $\mu_{1}$ is skew-symmetric and $\mu_{2}$ is symmetric, then $\left(A, \mu_{1}\right)$ is a Poisson algebra. 
2.3. Problems at issue. The first Rankin-Cohen bracket

$$
\mathrm{RC}_{1}(f, g)=k f \mathrm{D}(g)-\mathrm{D}(f) \ell g \quad\left(f \in \mathcal{M}_{k}, g \in M_{\ell}\right)
$$

gives $\mu_{*}$ a structure of Poisson algebra. This is a consequence of Corollary 4 . Cohen, Manin and Zagier [Cohen et al. 1997] and Yao [2007] (see also Rochberg, Tang and Yao [Rochberg et al. 2011]) proved that the family of Rankin-Cohen brackets is a formal deformation of $M_{*}$. In this case, the star product is called the Eholzer product. This subject has been widely studied. See for example [Olver and Sanders 2000; Pevzner 2008].

Can we construct formal deformations of $\mu_{*}^{\leq \infty}$ ? In other words, can we construct suitable families $\left(\mu_{n}\right)_{n \in \mathbb{N}}$ of bilinear maps on $\mu_{*}^{\leq \infty}$ that increase the weight by $2 n$, preserve the depth and define an analogue of the Eholzer product? The brackets defined in (1) do not lead to a solution since $\mathrm{RC}_{1}$ does not even provide $\mu_{*}^{\leq \infty}$ with a Poisson structure. Our first step is to obtain admissible Poisson brackets on $\mu_{*}^{\leq \infty}$ with the following definition.

Definition 5. A Poisson bracket $\mathrm{b}$ on $\mu_{*}^{\leq \infty}$ is admissible if

(1) $\mathrm{b}(f, g)=\mathrm{RC}_{1}(f, g)$ if $f$ and $g$ are in $M_{*}$;

(2) it satisfies $\mathrm{b}\left(\mathcal{M}_{k}^{\leq s}, M_{\ell}^{\leq t}\right) \subset \mathcal{M}_{k+\ell+2}^{\leq s+t}$ for all $k, \ell, s, t$.

Remark. We could have replaced condition (2) by the following one: there exists $e \geq 0$ such that $\mathrm{b}\left(\mathcal{M}_{k}^{\leq s}, \mu_{\ell}^{\leq t}\right) \subset \mathcal{M}_{k+\ell+e}^{\leq s+t}$ for all $k, \ell, s, t$. However, condition (1) implies that necessarily $e=2$.

Equivalently, a Poisson bracket $\mathrm{b}$ on $\mu_{*}^{\leq \infty}$ is admissible if and only if

$$
\begin{aligned}
\mathrm{b}\left(\mathrm{E}_{4}, \mathrm{E}_{6}\right) & =-2 \Delta, \\
\mathrm{b}\left(\mathrm{E}_{2}, \mathrm{E}_{4}\right) & \in M_{8}^{\leq \infty}, \quad \mathrm{b}\left(\mathrm{E}_{2}, \mathrm{E}_{6}\right) \in \mu_{10}^{\leq \infty}, \\
\mathrm{b}\left(\mathrm{E}_{2}, \mu_{*}\right) & \subset \mu_{*} \mathrm{E}_{2}+\mu_{*} .
\end{aligned}
$$

In order to classify the admissible Poisson brackets, we introduce the notion of Poisson modular isomorphism.

Definition 6. A Poisson isomorphism $\varphi:\left(\mu_{*}^{\leq \infty}, b_{1}\right) \rightarrow\left(\mu_{*}^{\leq \infty}, b_{2}\right)$ is called a Poisson modular isomorphism if $\varphi\left(M_{*}\right) \subset M_{*}$.

Indeed, if $\varphi$ is a Poisson modular isomorphism, then its restriction to the subalgebra $M_{*}$ is the identity. This is a consequence of the following proposition.

Proposition 7. The group of Poisson automorphisms of Poisson algebra $\left(\mu_{*}, \mathrm{RC}_{1}\right)$ is trivial. 
Proof. Let $\varphi$ be a Poisson automorphism of $M_{*}$. There exist two polynomials $s$ and $t$ in $\mathbb{C}[x, y]$ such that $\varphi\left(\mathrm{E}_{4}\right)=s\left(\mathrm{E}_{4}, \mathrm{E}_{6}\right)$ and $\varphi\left(\mathrm{E}_{6}\right)=t\left(\mathrm{E}_{4}, \mathrm{E}_{6}\right)$. By (2), we have

$$
\mathrm{RC}_{1}\left(\varphi\left(\mathrm{E}_{4}\right), \varphi\left(\mathrm{E}_{6}\right)\right)=\operatorname{jac}(s, t)\left(\mathrm{E}_{4}, \mathrm{E}_{6}\right) \cdot \mathrm{RC}_{1}\left(\mathrm{E}_{4}, \mathrm{E}_{6}\right) .
$$

Since $\varphi$ is an automorphism, $\operatorname{jac}(s, t)$ is a nonzero scalar, say $\lambda$. We get $\varphi\left(\mathrm{RC}_{1}\left(\mathrm{E}_{4}, \mathrm{E}_{6}\right)\right)=\lambda \mathrm{RC}_{1}\left(\mathrm{E}_{4}, \mathrm{E}_{6}\right)$ and hence $s^{3}-t^{2}=\lambda\left(x^{3}-y^{2}\right)$ in $\mathbb{C}[x, y]$.

We develop $s$ and $t$ into homogeneous components with respect to the weight:

where

$$
s=\sum_{\substack{i=0 \\ i \neq 1}}^{m} s_{2 i} \quad \text { and } \quad t=\sum_{\substack{i=0 \\ i \neq 1}}^{n} t_{2 i}
$$

$$
s_{2 i}=\sum_{\substack{(a, b) \in \mathbb{N}^{2} \\ 2 a+3 b=i}} \sigma_{a, b} x^{a} y^{b} \quad \text { and } \quad t_{2 i}=\sum_{\substack{(a, b) \in \mathbb{N}^{2} \\ 2 a+3 b=i}} \tau_{a, b} x^{a} y^{b} \quad\left(\sigma_{a, b}, \tau_{a, b} \in \mathbb{C}\right)
$$

for all $i$ (where $m=0$ or $m \geq 2$ and $n=0$ or $n \geq 2$ ). Equation (5) implies that $t\left(\mathrm{E}_{4}, \mathrm{E}_{6}\right)^{2}-s\left(\mathrm{E}_{4}, \mathrm{E}_{6}\right)^{3}$ has weight 12 . Then only three cases are possible.

(1) If $3 m>2 n$ then $m=2$ and so $n \in\{0,2\}$. This implies that $s=\sigma_{00}+\sigma_{10} x$ and $t=\tau_{00}+\tau_{10} x$. This contradicts $\operatorname{jac}(s, t) \neq 0$.

(2) If $3 m<2 n$ then $n=3$ and $m=0$. This contradicts jac $(s, t) \neq 0$.

(3) If $3 m=2 n$, we differentiate (5) with respect to $x$ and $y$ and get

$$
3 s^{2} \frac{\partial s}{\partial x}-2 t \frac{\partial t}{\partial x}=3 \lambda x^{2}, \quad 3 s^{2} \frac{\partial s}{\partial y}-2 t \frac{\partial t}{\partial y}=-2 \lambda y .
$$

This implies

$$
2 t=3 x^{2} \frac{\partial s}{\partial y}+2 y \frac{\partial s}{\partial x}, \quad 3 s^{2}=3 x^{2} \frac{\partial t}{\partial y}+2 y \frac{\partial t}{\partial x} .
$$

From the first differential equation of (6) we have

$$
2 t\left(\mathrm{E}_{4}, \mathrm{E}_{6}\right)=3 \mathrm{E}_{4}^{2} \frac{\partial s}{\partial y}\left(\mathrm{E}_{4}, \mathrm{E}_{6}\right)+2 \mathrm{E}_{6} \frac{\partial s}{\partial x}\left(\mathrm{E}_{4}, \mathrm{E}_{6}\right) .
$$

The highest weight of the right-hand side is less than or equal to $2 m+2$. This implies $n \leq m+1$. From the second differential equation of (6), we have

$$
3 s^{2}\left(\mathrm{E}_{4}, \mathrm{E}_{6}\right)=3 \mathrm{E}_{4}^{2} \frac{\partial t}{\partial y}\left(\mathrm{E}_{4}, \mathrm{E}_{6}\right)+2 \mathrm{E}_{6} \frac{\partial t}{\partial x}\left(\mathrm{E}_{4}, \mathrm{E}_{6}\right)
$$

hence $2 m \leq n+1$. We deduce $(m, n) \in\{(0,0),(2,3)\}$. Since $n=m=0$ would imply $\operatorname{jac}(s, t)=0$, we have $n=3$ and $m=2$. Then $s=\sigma_{00}+\sigma_{10} x$ and $t=\tau_{00}+\tau_{10} x+\tau_{01} y$. The first differential equation in (6) implies that $\tau_{00}=\tau_{10}=0$ and $\tau_{01}=\sigma_{10}$, whereas 
the second one implies that $\sigma_{00}=0$ and $\sigma_{10}=1$. Finally, $\varphi\left(\mathrm{E}_{4}\right)=s\left(\mathrm{E}_{4}, \mathrm{E}_{6}\right)=\mathrm{E}_{4}$ and $\varphi\left(\mathrm{E}_{6}\right)=s\left(\mathrm{E}_{4}, \mathrm{E}_{6}\right)=\mathrm{E}_{6}$.

Since $\mathrm{RC}_{1}(\Delta, f)=\left(12 \mathrm{D}(f)-k f \mathrm{E}_{2}\right) \Delta$ for any $f \in \mathcal{M}_{k}$, the first Rankin-Cohen bracket defines a derivation on $M_{*}$ called Serre's derivative by linear extension of

$$
\vartheta f=\frac{\mathrm{RC}_{1}(\Delta, f)}{12 \Delta}=\mathrm{D}(f)-\frac{k}{12} f \mathrm{E}_{2} \quad\left(f \in M_{k}\right) .
$$

This derivation is characterised by its values on the generators

$$
\vartheta \mathrm{E}_{4}=-\frac{1}{3} \mathrm{E}_{6}, \quad \vartheta \mathrm{E}_{6}=-\frac{1}{2} \mathrm{E}_{4}^{2} .
$$

We shall need the following result.

Proposition 8. The kernel of Serre's derivative is the Poisson centraliser of $\Delta$ for the first Rankin-Cohen bracket. This is $\mathbb{C}[\Delta]$.

Proof. If $f \in M_{k}$ is in $\operatorname{ker} \vartheta$ then $k f \mathrm{D}(\Delta)=12 \Delta \mathrm{D}(f)$. Solving the differential equation, we find that 12 divides $k$ and that $f \in \mathbb{C} \Delta^{k / 12}$.

We note that for any $g \in \mathcal{M}_{\ell}$ we have

$$
\mathrm{RC}_{1}\left(\Delta^{m}, g\right)=m \Delta^{m}\left(12 \mathrm{D}(g)-\ell g \mathrm{E}_{2}\right)
$$

and deduce that for any $f \in \mathbb{C}[\Delta]$ and $g \in \mathcal{M}_{*}$ we have

$$
\mathrm{RC}_{1}(f, g)=12 \xi(f) \vartheta(g),
$$

where $\xi$ is the Eulerian derivative on $\mathbb{C}[\Delta]$ defined by $\xi=\Delta \frac{\partial}{\partial \Delta}$.

\section{Poisson structures on quasimodular forms}

3.1. First family. This section is devoted to the proof of Proposition A.

We fix $\lambda \in \mathbb{C}^{*}$ and introduce in $\mathbb{C}[x, y, z]$ the three polynomials

$$
\begin{aligned}
& r(x, y, z)=\frac{1}{3}\left(\lambda y^{2}-2 x z\right), \\
& p(x, y, z)=-2\left(y^{3}-z^{2}\right), \\
& q(x, y, z)=-\frac{1}{2}\left(\lambda y z-2 x y^{2}\right) .
\end{aligned}
$$

Since $(p, q, r) \cdot \operatorname{curl}(p, q, r)=0$, we define a Poisson bracket on $\mu_{*}^{\leq \infty}$ if we set

$$
\begin{aligned}
& \left\{\mathrm{E}_{4}, \mathrm{E}_{6}\right\}_{\lambda}=p\left(\mathrm{E}_{2}, \mathrm{E}_{4}, \mathrm{E}_{6}\right), \\
& \left\{\mathrm{E}_{2}, \mathrm{E}_{4}\right\}_{\lambda}=r\left(\mathrm{E}_{2}, \mathrm{E}_{4}, \mathrm{E}_{6}\right), \\
& \left\{\mathrm{E}_{6}, \mathrm{E}_{2}\right\}_{\lambda}=q\left(\mathrm{E}_{2}, \mathrm{E}_{4}, \mathrm{E}_{6}\right) .
\end{aligned}
$$


Let us prove that $\{,\}_{\lambda}$ is not unimodular. If it were, we would have $k \in \mathbb{C}[x, y, z]$ such that $\operatorname{curl}(p, q, r)=(p, q, r) \wedge \operatorname{grad} k$. Identifying the first components would lead to

$$
\frac{7}{6} \lambda y=\frac{1}{2}\left(-\lambda y z+2 y^{2} x\right) \frac{\partial k}{\partial z}-\frac{1}{3}\left(\lambda y^{2}-2 z x\right) \frac{\partial k}{\partial y},
$$

which has no solution in $\mathbb{C}[x, y, z]$.

A Poisson modular isomorphism $\varphi_{\lambda}$ between $\left(\mathcal{M}_{*}^{\leq \infty},\{,\}_{\lambda}\right)$ and $\left(\mathcal{M}_{*}^{\leq \infty},\{,\}_{1}\right)$ is determined by

$$
\varphi_{\lambda}\left(\mathrm{E}_{2}\right)=\lambda \mathrm{E}_{2}, \quad \varphi_{\lambda}\left(\mathrm{E}_{4}\right)=\mathrm{E}_{4}, \quad \varphi_{\lambda}\left(\mathrm{E}_{6}\right)=\mathrm{E}_{6} .
$$

Finally, we determine the Poisson centre of the Poisson algebra $\left(\mu_{*}^{\leq \infty},\{,\}_{1}\right)$. Let us define a derivation on $M_{*}$ by $\sigma=2 \vartheta$ (see (7)) and a derivation on $M_{*}$ by linear extension of

$$
\delta(f)=\frac{k}{12} f \mathrm{E}_{4} \quad\left(f \in \mathcal{M}_{k}\right) .
$$

We note that $\left(\mu_{*}^{\leq \infty},\{,\}_{1}\right)$ is the Poisson-Ore extension $\mathbb{C}\left[\mathrm{E}_{4}, \mathrm{E}_{6}\right]\left[\mathrm{E}_{2}\right]_{\sigma, \delta}$. Now consider any $f \in M_{*}^{\leq \infty}$ written as

$$
f=\sum_{i=0}^{s} f_{i} \mathrm{E}_{2}^{i} \quad\left(f_{i} \in \mathcal{M}_{*}\right)
$$

We compute

$$
\left\{\mathrm{E}_{2}, f\right\}_{1}=\delta\left(f_{0}\right)+\sum_{i=1}^{s}\left(\sigma\left(f_{i-1}\right)+\delta\left(f_{i}\right)\right) \mathrm{E}_{2}^{i}+\sigma\left(f_{s}\right) \mathrm{E}_{2}^{s+1} .
$$

If $\left\{\mathrm{E}_{2}, f\right\}_{1}=0$ then $\delta\left(f_{0}\right)=0$, and hence $f_{0} \in \mathbb{C}$ and $\sigma\left(f_{0}\right)=0$. We obtain inductively that $f_{i} \in \mathbb{C}$ for all $0 \leq i \leq s$, so the Poisson centraliser of $\mathrm{E}_{2}$ is $\mathbb{C}\left[\mathrm{E}_{2}\right]$. Suppose that the Poisson centre contains a nonscalar element. Then it is in the Poisson centraliser of $\mathrm{E}_{2}$ and can be written

$$
f=\sum_{j=0}^{p} \alpha_{j} \mathrm{E}_{2}^{j} \quad\left(p \geq 1, \alpha_{j} \in \mathbb{C}, \alpha_{p} \neq 0\right) .
$$

We compute

$$
\left\{\mathrm{E}_{4}, f\right\}_{1}=\sum_{j=0}^{p} j \alpha_{j} \mathrm{E}_{2}^{j-1} \cdot\left\{\mathrm{E}_{4}, \mathrm{E}_{2}\right\}_{1}
$$

and find that the coefficient of $\mathrm{E}_{2}^{p}$ is nonzero. It follows that $f$ is not in the Poisson centre. 
3.2. Second family. This section is devoted to the proof of Proposition C. We fix $\alpha \in \mathbb{C}$ and introduce in $\mathbb{C}[x, y, z]$ the three polynomials

$$
\begin{aligned}
& r(x, y, z)=\alpha x z, \\
& p(x, y, z)=-2\left(y^{3}-z^{2}\right), \\
& q(x, y, z)=-\frac{3}{2} \alpha x y^{2} .
\end{aligned}
$$

Since $(p, q, r) \cdot \operatorname{curl}(p, q, r)=0$, we define a Poisson bracket on $\mu_{*}^{\leq \infty}$ if we set

$$
\begin{aligned}
& \left(\mathrm{E}_{4}, \mathrm{E}_{6}\right)_{\alpha}=p\left(\mathrm{E}_{2}, \mathrm{E}_{4}, \mathrm{E}_{6}\right), \\
& \left(\mathrm{E}_{2}, \mathrm{E}_{4}\right)_{\alpha}=r\left(\mathrm{E}_{2}, \mathrm{E}_{4}, \mathrm{E}_{6}\right), \\
& \left(\mathrm{E}_{6}, \mathrm{E}_{2}\right)_{\alpha}=q\left(\mathrm{E}_{2}, \mathrm{E}_{4}, \mathrm{E}_{6}\right) .
\end{aligned}
$$

Assume $\alpha \neq 4$. Let us prove that $(,)_{\alpha}$ is not unimodular. If it were, we would have $k \in \mathbb{C}[x, y, z]$ such that $\operatorname{curl}(p, q, r)=(p, q, r) \wedge \operatorname{grad} k$. Identifying the second components would lead to

$$
(4-\alpha) z=\alpha x z \frac{\partial k}{\partial x}+2\left(y^{3}-z^{2}\right) \frac{\partial k}{\partial z},
$$

which has no solution in $\mathbb{C}[x, y, z]$.

If $\alpha=4$, then $(p, q, r)=\operatorname{grad} k_{0}$, where $k_{0}=-2\left(y^{3}-z^{2}\right) x$. As a consequence, the bracket $(,)_{4}$ provides $\mu_{*}^{\leq \infty}$ with a Jacobian Poisson structure of potential $k_{0}=-2 \Delta \mathrm{E}_{2}=-2 \mathrm{D}(\Delta)$.

If $\varphi:\left(M_{*}^{\leq \infty},(,)_{\alpha}\right) \rightarrow\left(M_{*}^{\leq \infty},(,)_{\alpha^{\prime}}\right)$ is a Poisson modular isomorphism, let us prove that $\alpha=\alpha^{\prime}$. By Proposition 7 , we have $\varphi\left(\mathrm{E}_{4}\right)=\mathrm{E}_{4}$ and $\varphi\left(\mathrm{E}_{6}\right)=\mathrm{E}_{6}$. By surjectivity, it follows that $\varphi\left(\mathrm{E}_{2}\right)=\eta \mathrm{E}_{2}+F$ for some $\eta \in \mathbb{C}^{*}$ and $F \in \mathcal{M}_{*}$. We compute

$$
\varphi\left(\left(\mathrm{E}_{2}, \mathrm{E}_{4}\right)_{\alpha}\right)=\alpha \eta \mathrm{E}_{6} \mathrm{E}_{2}+\alpha \mathrm{E}_{6} F
$$

and

$$
\left(\varphi\left(\mathrm{E}_{2}\right), \varphi\left(\mathrm{E}_{4}\right)\right)_{\alpha^{\prime}}=\alpha^{\prime} \eta \mathrm{E}_{6} \mathrm{E}_{2}+\left(F, \mathrm{E}_{4}\right)_{\alpha^{\prime}} .
$$

Since $\left(F, \mathrm{E}_{4}\right)_{\alpha^{\prime}} \in M_{*}$ we get $\alpha^{\prime}=\alpha$.

Finally, we determine the Poisson centre of the Poisson algebra $\left(\mu_{*}^{\leq \infty},(,)_{\alpha}\right)$. We note that $\left(\mu_{*}^{\leq \infty},(,)_{\alpha}\right)$ is the Poisson-Ore extension $\mathbb{C}\left[\mathrm{E}_{4}, \mathrm{E}_{6}\right]\left[\mathrm{E}_{2}\right]_{\sigma, \delta}$, where $\sigma=-3 \alpha \vartheta($ see $(7))$ and $\delta=0$. Let

$$
f=\sum_{j=0}^{s} f_{j} \mathrm{E}_{2}^{j} \quad\left(f_{j} \in M_{*}\right) .
$$

We have

$$
\left(\mathrm{E}_{2}, f\right)_{\alpha}=\sum_{j=0}^{s} \sigma\left(f_{j}\right) \mathrm{E}_{2}^{j+1}
$$


and hence $f$ is in the Poisson centraliser of $\mathrm{E}_{2}$ if and only if each $f_{j}$ is in the Poisson centraliser of $\Delta$ for $\mathrm{RC}_{1}$. By Proposition 8, we deduce that the centraliser of $\mathrm{E}_{2}$ is $\mathbb{C}\left[\Delta, \mathrm{E}_{2}\right]$. Let

$$
f=\sum_{j=0}^{s} f_{j}(\Delta) \mathrm{E}_{2}^{j} \in \mathbb{C}\left[\Delta, \mathrm{E}_{2}\right] .
$$

We use (8) to compute

$$
\begin{aligned}
& \left(f, \mathrm{E}_{4}\right)_{\alpha}=\sum_{j=0}^{s}\left(-4 \xi\left(f_{j}\right)+j \alpha f_{j}\right) \mathrm{E}_{6} \mathrm{E}_{2}^{j}, \\
& \left(f, \mathrm{E}_{6}\right)_{\alpha}=\frac{3}{2} \sum_{j=0}^{s}\left(-4 \xi\left(f_{j}\right)+j \alpha f_{j}\right) \mathrm{E}_{4}^{2} \mathrm{E}_{2}^{j} .
\end{aligned}
$$

We deduce that $f$ is in the Poisson centre of $(,)_{\alpha}$ if and only if

$$
\xi\left(f_{j}\right)=\frac{j \alpha}{4} f_{j}
$$

for all $j$, that is, if and only if any $f_{j}$ is of the form $f_{j}=\lambda_{j} \Delta^{m_{j}}$ for some $\lambda_{j} \in \mathbb{C}$ and $m_{j} \in \mathbb{N}$ such that $j \alpha=4 m_{j}$. If $\alpha \notin \mathbb{Q}$ or if $\alpha<0$ then $j=0$ and $m_{j}=0$, and hence $f=f_{0} \in \mathbb{C}$. If $\alpha=p / q$ with $p \geq 1, q \geq 1$ and $(p, q)=1$, then $\lambda \Delta^{m_{j}} \mathrm{E}_{2}^{j}$ is in the Poisson centre if and only if $p j=4 q m_{j}$. The result follows by obvious arithmetical consideration. Finally, if $\alpha=0$, then $(,)_{0}$ is the trivial bracket and its Poisson centre is $\mathbb{C}\left[\mathrm{E}_{2}\right]$.

3.3. Third family. In this section, we study the third family, that is, we prove Proposition E.

For any $\mu \in \mathbb{C}$, let us introduce

$$
k_{\mu}=-2 \Delta \mathrm{E}_{2}+\mu \mathrm{E}_{4}^{2} \mathrm{E}_{6} .
$$

Then

$$
\begin{aligned}
& \operatorname{jac}\left(\mathrm{E}_{4}, \mathrm{E}_{6}, k_{\mu}\right)=\frac{\partial k_{\mu}}{\partial \mathrm{E}_{2}}=-2 \mathrm{E}_{4}^{3}+2 \mathrm{E}_{6}^{2}, \\
& \operatorname{jac}\left(\mathrm{E}_{2}, \mathrm{E}_{4}, k_{\mu}\right)=\frac{\partial k_{\mu}}{\partial \mathrm{E}_{6}}=4 \mathrm{E}_{6} \mathrm{E}_{2}+\mu \mathrm{E}_{4}^{2}, \\
& \operatorname{jac}\left(\mathrm{E}_{2}, \mathrm{E}_{6}, k_{\mu}\right)=-\frac{\partial k_{\mu}}{\partial \mathrm{E}_{4}}=6 \mathrm{E}_{4}^{2} \mathrm{E}_{2}-2 \mu \mathrm{E}_{4} \mathrm{E}_{6} .
\end{aligned}
$$

The third family of Poisson brackets is then defined by $\langle f, g\rangle_{\mu}=\operatorname{jac}\left(f, g, k_{\mu}\right)$. With the notation of Proposition $\mathrm{C}$, we have in particular $\langle f, g\rangle_{0}=(f, g)_{4}$.

For any $\mu \in \mathbb{C}^{*}$, define a Poisson modular isomorphism $\varphi_{\mu}$ between $\left(\mu_{*}^{\leq \infty},\langle,\rangle_{\mu}\right)$ and $\left(\mu_{*}^{\leq \infty},\langle,\rangle_{1}\right)$ by setting $\varphi_{\mu}\left(\mathrm{E}_{2}\right)=\mu \mathrm{E}_{2}, \varphi_{\mu}\left(\mathrm{E}_{4}\right)=\mathrm{E}_{4}$ and $\varphi_{\mu}\left(\mathrm{E}_{6}\right)=\mathrm{E}_{6}$. 
Since the degree in $\mathrm{E}_{2}$ of $k_{\mu}$ as a polynomial in $\mathrm{E}_{2}, \mathrm{E}_{4}, \mathrm{E}_{6}$ is 1 , Lemma 1 implies that the Poisson centre of $\left(\mu_{*}^{\leq \infty},\langle,\rangle_{\mu}\right)$ is $\mathbb{C}\left[k_{\mu}\right]$.

3.4. Classification. This section is devoted to the proof of Theorem F.

Let $\{$,$\} be an admissible bracket on \mu_{*}^{\leq \infty}$. By Definition 5 and Theorem 2, there exist a Poisson derivation $\sigma$ of $\mu_{*}$ and a Poisson $\sigma$-derivation $\delta$ of $\mu_{*}$ such that

$$
\left\{\mathrm{E}_{2}, f\right\}=\sigma(f) \mathrm{E}_{2}+\delta(f) \quad\left(f \in \mathcal{M}_{*}\right) .
$$

By definition, $\sigma\left(M_{k}\right) \subset M_{k+2}$ and $\delta\left(\mathcal{M}_{k}\right) \subset \mathcal{M}_{k+4}$ for any $k$. The admissible bracket $\{$,$\} is then defined by the four scalars \alpha, \beta, \gamma$ and $\varepsilon$ such that

$$
\sigma\left(\mathrm{E}_{4}\right)=\alpha \mathrm{E}_{6}, \quad \delta\left(\mathrm{E}_{4}\right)=\beta \mathrm{E}_{4}^{2}, \quad \sigma\left(\mathrm{E}_{6}\right)=\gamma \mathrm{E}_{4}^{2} \quad \text { and } \quad \delta\left(\mathrm{E}_{6}\right)=\varepsilon \mathrm{E}_{4} \mathrm{E}_{6} .
$$

The condition that $\sigma$ is a Poisson derivation imposes the condition

$$
\left\{\sigma\left(\mathrm{E}_{4}\right), \mathrm{E}_{6}\right\}+\left\{\mathrm{E}_{4}, \sigma\left(\mathrm{E}_{6}\right)\right\}=-2 \sigma\left(\mathrm{E}_{4}^{3}-\mathrm{E}_{6}^{2}\right),
$$

or equivalently, $3 \alpha=2 \gamma$. The condition that $\delta$ is a Poisson $\sigma$-derivation imposes

$$
\delta\left(\left\{\mathrm{E}_{4}, \mathrm{E}_{6}\right\}\right)=(2 \beta+\varepsilon) \mathrm{E}_{4}\left\{\mathrm{E}_{4}, \mathrm{E}_{6}\right\}+\alpha \varepsilon \mathrm{E}_{4} \mathrm{E}_{6}^{2}-\beta \gamma \mathrm{E}_{4}^{4},
$$

or equivalently,

$$
\left\{\begin{array}{l}
4 \beta+(\alpha-2) \varepsilon=0 \\
(3 \alpha-4) \beta+4 \varepsilon=0 .
\end{array}\right.
$$

Either $\beta=\varepsilon=0$ is the only solution, or $\alpha \in\left\{-\frac{2}{3}, 4\right\}$ and $\varepsilon=\frac{4}{2-\alpha} \beta$.

- The case $\beta=\varepsilon=0$ leads to the second family: $\{\}=,(,)_{\alpha}$.

- The case $\alpha=-\frac{2}{3}$ and $\varepsilon=3 \beta / 2 \neq 0$ leads to the first family: $\{\}=,\{,\}_{3 \beta}$.

- The case $\alpha=4$ and $\varepsilon=-2 \beta \neq 0$ leads to the third family: $\{\}=,\langle,\rangle_{\beta}$.

Using Propositions $\mathrm{C}$ and $\mathrm{E}$, we conclude that the only admissible Poisson brackets, up to Poisson modular isomorphisms, are $\{,\}_{1},\langle,\rangle_{1}$ and $(,)_{\alpha}$ for any $\alpha \in \mathbb{C}$. Looking at the centres, it is clear that the Poisson algebras $\left(\mu_{*}^{\leq \infty},\langle,\rangle_{1}\right)$ and $\left(M_{*}^{\leq \infty},\{,\}_{1}\right)$ are not Poisson modular isomorphic. Suppose that there exists a Poisson modular isomorphism $\varphi$ from $\left(M_{*}^{\leq \infty},(,)_{\alpha}\right)$ to $\left(M_{*}^{\leq \infty},\{,\}_{1}\right)$. We know (see Section 3.2) that

$$
\varphi\left(\mathrm{E}_{4}\right)=\mathrm{E}_{4}, \quad \varphi\left(\mathrm{E}_{6}\right)=\mathrm{E}_{6} \quad \text { and } \quad \varphi\left(\mathrm{E}_{2}\right)=\eta \mathrm{E}_{2}+F
$$

for some $\eta \in \mathbb{C}^{*}$ and $F \in \mathcal{M}_{*}$. From $\varphi\left(\left(\mathrm{E}_{2}, \mathrm{E}_{4}\right)_{\alpha}\right)=\left\{\varphi\left(\mathrm{E}_{2}\right), \varphi\left(\mathrm{E}_{4}\right)\right\}_{1}$ we obtain

$$
\alpha \eta \mathrm{E}_{6} \mathrm{E}_{2}+\alpha F \mathrm{E}_{6}=-\frac{2}{3} \eta \mathrm{E}_{6} \mathrm{E}_{2}+\frac{1}{3} \eta \mathrm{E}_{4}^{2}+\left\{F, \mathrm{E}_{4}\right\}_{1},
$$

and hence

$$
\alpha=-\frac{2}{3}, \quad \frac{1}{3} \eta \mathrm{E}_{4}^{2}=-\frac{2}{3} F \mathrm{E}_{6}-\left\{F, \mathrm{E}_{4}\right\}_{1}=-\frac{2}{3} F \mathrm{E}_{6}+2\left(\mathrm{E}_{4}^{3}-\mathrm{E}_{6}^{2}\right) \frac{\partial F}{\partial \mathrm{E}_{6}}
$$


by (2). We get a contradiction. Replacing $\{,\}_{1}$ by $\langle,\rangle_{1}$, we get

$$
\alpha=4, \quad \eta \mathrm{E}_{4}^{2}=4 F \mathrm{E}_{6}-2\left(\mathrm{E}_{4}^{3}-\mathrm{E}_{6}^{2}\right) \frac{\partial F}{\partial \mathrm{E}_{6}},
$$

and again we get a contradiction.

\section{Star products on quasimodular forms}

4.1. Extension of the first family. This section is devoted to proving Theorem B. We will use the following result of Zagier [1994, Example 1]. Let $A=\bigoplus A_{k}$ be a commutative graded algebra with a derivation $d$ homogeneous of degree 2 (that is, $\left.\mathrm{d}\left(A_{k}\right) \subset A_{k+2}\right)$. Let us define, for any $f \in A_{k}, g \in A_{\ell}, r \geq 0$ :

$$
[f, g]_{d, r}=\sum_{i=0}^{r}(-1)^{i}\left(\begin{array}{c}
k+r-1 \\
r-i
\end{array}\right)\left(\begin{array}{c}
\ell+r-1 \\
i
\end{array}\right) \mathrm{d}^{i}(f) \mathrm{d}^{r-i}(g) \in A_{k+\ell+2 r} .
$$

Then $A$ equipped with these brackets is a Rankin-Cohen algebra, which means that all algebraic identities satisfied by the usual Rankin-Cohen brackets on modular forms are also satisfied, in particular those expressing the associativity of the corresponding star product. We obtain the following result.

Theorem 9. The star product defined by

$$
f \# g=\sum_{n \geq 0}[f, g]_{\mathrm{d}, n} \hbar^{n}
$$

defines a formal deformation on A.

In particular, we recover the fact, given by Corollary 4 , that $[,]_{\mathrm{d}, 1}$ is a Poisson bracket. Note also that this theorem can be obtained from Connes and Moscovici's result cited below (see Section 4.2).

Let $a \in \mathbb{C}$ and $\mathrm{d}_{a}$ be the homogeneous derivation of degree 2 on $\mu_{*}^{\leq \infty}$ defined by

$$
\mathrm{d}_{a}\left(\mathrm{E}_{2}\right)=2 a \mathrm{E}_{2}^{2}-\frac{1}{12} \mathrm{E}_{4}, \quad \mathrm{~d}_{a}\left(\mathrm{E}_{4}\right)=4 a \mathrm{E}_{4} \mathrm{E}_{2}-\frac{1}{3} \mathrm{E}_{6}, \quad \mathrm{~d}_{a}\left(\mathrm{E}_{6}\right)=6 e \mathrm{E}_{6} \mathrm{E}_{2}-\frac{1}{2} \mathrm{E}_{4}^{2} .
$$

A direct computation proves that the two Poisson brackets $[,]_{\mathrm{d}_{a}, 1}$ and $\{,\}_{1}$ coincide on generators and hence are equal on $\mu_{*}^{\leq \infty}$.

Remark. A derivation $\mathrm{d}$ on $\mu_{*}^{\leq \infty}$ is complex-like if $\mathrm{d} \mu_{k}^{\leq s} \subset \mu_{k+2}^{\leq s+1}$ for all $k$ and $s$. Let $\pi$ be the derivation on $\mu_{*}^{\leq \infty}$ defined by linear extension of $\pi(f)=k f \mathrm{E}_{2}$ for all $f \in \mathcal{M}_{k}^{\leq \infty}$. The set of complex-like derivations d such that $[,]_{\mathrm{d}, 1}=0$ is the vector space of dimension 1 over $\mathbb{C}$ generated by $\pi$. Let us define $\mathrm{w}$ on $\mu_{*}^{\leq \infty}$ by

Then

$$
\mathrm{w}(f)=\frac{\{\Delta, f\}_{1}}{12 \Delta} .
$$

$$
\mathrm{d}_{a}=\mathrm{w}+a \delta .
$$


This implies in particular that if a complex-like derivation $\mathrm{d}$ satisfies $[,]_{\mathrm{d}, 1}=\{,\}_{1}$, then $\mathrm{d}=\mathrm{d}_{a}$ for some $a \in \mathbb{C}$.

Point (ii) of Theorem B is obtained by a direct application of Theorem 9. We prove now (iii). The term of highest degree with respect to $\mathrm{E}_{2}$ in $\left[\mathrm{E}_{2}, \mathrm{E}_{4}\right]_{\mathrm{d}_{a}, 2}$ is $8 a^{2} \mathrm{E}_{4} \mathrm{E}_{2}^{3}$. This forces $a=0$. Conversely, if $a=0$, then $\mathrm{d}_{0} M_{*}^{\leq \infty} \subset M_{*}$. For any $f=f_{i} \mathrm{E}_{2}^{i}$ with $f_{i} \in \mathcal{M}_{*}$, we have

$$
\mathrm{d}_{0}(f)=\mathrm{d}_{0}\left(f_{i}\right) \mathrm{E}_{2}^{i}-\frac{1}{12} i f_{i} \mathrm{E}_{4} \mathrm{E}_{2}^{i-1},
$$

and hence $\operatorname{deg}_{\mathrm{E}_{2}} \mathrm{~d}_{0}(f) \leq \operatorname{deg}_{\mathrm{E}_{2}} f$ and $\operatorname{deg}_{\mathrm{E}_{2}} \mathrm{~d}_{0}^{j}(f) \leq \operatorname{deg}_{\mathrm{E}_{2}} f$ for any $f \in \mathcal{M}_{*}^{\leq \infty}$ and $j \geq 0$. This implies that

$$
\left[\mathcal{M}_{k}^{\leq s}, \mathcal{M}_{\ell}^{\leq t}\right]_{\mathrm{d}_{0}, n} \subset \mathcal{M}_{k+\ell+2 n}^{\leq s+t} .
$$

4.2. Extension of the second family. The aim of this section is to prove Theorem $D$. The proof of (i) is similar to the proof of (i) in Theorem B. Let $\mathscr{K}: \mu_{*}^{\leq \infty} \rightarrow \mathbb{C}$ be a graded-additive map. For any integer $n \geq 0$, we define a bilinear application $[,]_{\mathrm{d}, n}^{\mathscr{K}}$ by bilinear extension of

$$
[f, g]_{\mathrm{d}, n}^{\mathscr{K}}=\sum_{r=0}^{n}(-1)^{r}\left(\begin{array}{c}
\mathscr{K}(f)+n-1 \\
n-r
\end{array}\right)\left(\begin{array}{c}
\mathscr{K}(g)+n-1 \\
r
\end{array}\right) \mathrm{d}^{r} f \mathrm{~d}^{n-r} g .
$$

By Corollary 4 , we know that $[f, g]_{\mathrm{d}, 1}^{\mathscr{K}}$ is a Poisson bracket.

Let us fix $\mathscr{K}_{\alpha}$ to be the linear extension on $\mathcal{M}_{*}^{\leq \infty}=\bigoplus_{k} \bigoplus_{s} \mathcal{M}_{k}^{s}$ of

$$
\mathscr{K}_{\alpha}(f)=(k-(3 \alpha+2) s) \quad\left(f \in \mathcal{M}_{k}^{s}\right) .
$$

Let $\pi_{\alpha}$ be the derivation on $\mathcal{M}_{*}^{\leq \infty}$ defined by $\pi_{\alpha}(f)=\mathscr{K}_{\alpha}(f) f \mathrm{E}_{2}$ for all $f \in \mathcal{M}_{*}^{\leq \infty}$. The set of complex-like derivations such that $[,]_{\mathrm{d}, 1}^{\mathscr{K}_{\alpha}}=0$ is the vector space of dimension 1 over $\mathbb{C}$ generated by $\pi_{\alpha}$. Define derivations $\mathrm{v}$ and $\delta_{\alpha, b}$ on $\mu_{*}^{\leq \infty}$ by

and

$$
\mathrm{v}(f)=\frac{(\Delta, f)_{\alpha}}{12 \Delta}
$$

$$
\delta_{\alpha, b}=\mathrm{v}+b \pi_{\alpha} .
$$

Note that $\mathrm{v}$ does not depend on $\alpha$. By comparing the values on the generators, it is immediate that $(,)_{\alpha}=[,]_{\delta_{\alpha, b}, 1}^{\mathscr{K}_{\alpha}}$.

Remark. Direct computations show that if $\mathrm{d}$ is a homogeneous derivation of degree 2 and $\mathscr{K}$ is such that $(,)_{\alpha}=[,]_{\mathrm{d}, 1}^{\mathscr{K}}$, then we necessarily have $\mathscr{K}=\mathscr{K}_{\alpha}$ and $\mathrm{d}=\delta_{\alpha, b}$ for some $b \in \mathbb{C}$.

The condition that $\left[\mathrm{E}_{4}, \mathrm{E}_{6}\right]_{\delta_{\alpha, b}, 2}^{\mathscr{\alpha}}$ has to be a modular form implies $b=0$ or $\alpha=-\frac{1}{3}$. For $\alpha=-\frac{1}{3}$, condition (4) for $\mu_{r}=[,]_{\delta_{\alpha, b}, r}^{\mathscr{K}}$ and $n=3$ is not satisfied 
(this can be shown with computer assistance, for example with Sage [Stein et al. 2013]). We assume then that $b=0$.

Connes and Moscovici [2004, Remark 14] (see also [Yao 2007, §II.2] for a nice presentation of this result) proved that if $E$ and $H$ are two derivations of an algebra $R$ such that $H E-E H=E$, then the applications $\mu_{n}: R \times R \rightarrow R$ defined by

$$
\mu_{n}(f, g)=\sum_{r=0}^{n} \frac{(-1)^{r}}{r !(n-r) !}\left[E^{r} \circ(2 H+r)^{\langle n-r\rangle}(f)\right] \cdot\left[E^{n-r} \circ(2 H+n-r)^{\langle r\rangle}(g)\right]
$$

define a formal deformation on $R$ with the notation

$$
F^{\langle m\rangle}=F \circ(F+1) \circ(F+2) \circ \cdots \circ(F+m-1) .
$$

Let $\varpi$ be the derivation defined on $\mu_{*}^{\leq \infty}$ by $\varpi(f)=\mathscr{K}(f) f$. Then we have

$$
\varpi \circ \delta_{\alpha, 0}-\delta_{\alpha, 0} \circ \varpi=2 \delta_{\alpha, 0} .
$$

We use Connes and Moscovici's result with $E=\delta_{\alpha, 0}$ and $H=\varpi / 2$ to obtain $\mu_{n}(f, g)$

$$
=\sum_{r=0}^{n}(-1)^{r}\left(\begin{array}{c}
k-(3 \alpha+2) s+n-1 \\
n-r
\end{array}\right)\left(\begin{array}{c}
\ell-(3 \alpha+2) t+n-1 \\
r
\end{array}\right) \delta_{\alpha, 0}^{r}(f) \delta_{\alpha, 0}^{n-r}(g) .
$$

This implies Theorem D.

Remark. We could have applied Connes and Moscovici's result to extend the first family. Indeed Zagier's result is a consequence of Connes and Moscovici's. Let $d$ be a derivation homogeneous of degree 2 of the commutative graded algebra $A=\bigoplus A_{k}$. It is obvious that the linear map defined on each $A_{k}$ by $H(f)=(k / 2) f$ is a derivation of $A$. It is also clear that it satisfies $H \circ d-d \circ H=d$. In particular, for any $f \in A_{k}$ and $g \in A_{\ell}$ we calculate

$$
\begin{aligned}
(2 H+r)^{\langle n-r\rangle}(f) & =\frac{(k+n-1) !}{(k+r-1) !} f, \\
(2 H+(n-r))^{\langle r\rangle}(g) & =\frac{(\ell+n-1) !}{(\ell+n-r-1) !} g .
\end{aligned}
$$

Hence a direct application of formula (11) gives formula (9).

4.3. Extension of the third family. We do not extend the third family, since for $\mu \neq 0$, the bracket $\langle,\rangle_{\mu}$ does not have the shape of a Rankin-Cohen bracket. More precisely, if there exist a function $\kappa: \mathcal{M}_{*}^{\leq \infty} \rightarrow \mathbb{C}$ and a complex-like derivation $\delta$ of $\mu_{*}^{\leq \infty}$ such that

$$
\langle f, g\rangle_{\mu}=\kappa(f) f \delta(g)-\kappa(g) g \delta(f)
$$


for all $f$ and $g$ in $\mu_{*}^{\leq \infty}$, then $\mu=0$. Indeed, assume $\kappa$ and $\delta$ exist; then

$$
\left\{\begin{array}{l}
\delta\left(\mathrm{E}_{2}\right)=A \mathrm{E}_{2}^{2}+B \mathrm{E}_{4}, \\
\delta\left(\mathrm{E}_{4}\right)=C \mathrm{E}_{4} \mathrm{E}_{2}+D \mathrm{E}_{6}, \\
\delta\left(\mathrm{E}_{6}\right)=E \mathrm{E}_{6} \mathrm{E}_{2}+F \mathrm{E}_{4}^{2}
\end{array}\right.
$$

for some complex numbers $A, B, C, D, E$ and $F$. Since we know the values of $\langle,\rangle_{\mu}$ on the generators, we get a system depending on $A, B, C, D, E, F, \kappa\left(\mathrm{E}_{2}\right)$, $\kappa\left(\mathrm{E}_{4}\right)$ and $\kappa\left(\mathrm{E}_{6}\right)$. It is not difficult to prove that this system has a solution if and only if $\mu=0$.

\section{References}

[Bieliavsky et al. 2007] P. Bieliavsky, X. Tang, and Y.-J. Yao, "Rankin-Cohen brackets and formal quantization”, Adv. Math. 212:1 (2007), 293-314. MR 2008f:53125 Zbl 1123.53049

[Cohen 1975] H. Cohen, "Sums involving the values at negative integers of $L$-functions of quadratic characters", Math. Ann. 217:3 (1975), 271-285. MR 52 \#3080 Zbl 0311.10030

[Cohen et al. 1997] P. B. Cohen, Y. Manin, and D. Zagier, "Automorphic pseudodifferential operators", pp. 17-47 in Algebraic aspects of integrable systems: in memory of Irene Dorfman, edited by A. S. Fokas and I. M. Gelfand, Progr. Nonlinear Differential Equations Appl. 26, Birkhäuser, Boston, 1997. MR 98e:11054 Zbl 1055.11514

[Connes and Moscovici 2004] A. Connes and H. Moscovici, "Rankin-Cohen brackets and the Hopf algebra of transverse geometry", Mosc. Math. J. 4:1 (2004), 111-130. MR 2005f:11079b Zbl 1122.11024

[Dufour and Haraki 1991] J.-P. Dufour and A. Haraki, "Rotationnels et structures de Poisson quadratiques”, C. R. Acad. Sci. Paris Sér. I Math. 312:1 (1991), 137-140. MR 92a:53045 Zbl 0719.58001

[Gutierrez and Sevilla 2006] J. Gutierrez and D. Sevilla, "Computation of unirational fields", J. Symbolic Comput. 41:11 (2006), 1222-1244. MR 2007g:12003 Zbl 1149.12001

[Kobayashi and Pevzner 2013] T. Kobayashi and M. Pevzner, "Rankin-Cohen operators for symmetric pairs", preprint, 2013. arXiv 1301.2111

[Laurent-Gengoux et al. 2013] C. Laurent-Gengoux, A. Pichereau, and P. Vanhaecke, Poisson structures, Grundlehren der Mathematischen Wissenschaften 347, Springer, Heidelberg, 2013. MR 2906391 Zbl 1271.53074

[Martin and Royer 2005] F. Martin and E. Royer, "Formes modulaires et périodes", pp. 1-117 in Formes modulaires et transcendance (Marseille, 2003), edited by S. Fischler et al., Sémin. Congr. 12, Soc. Math. France, Paris, 2005. MR 2007a:11065 Zbl 1104.11017

[Martin and Royer 2009] F. Martin and E. Royer, "Rankin-Cohen brackets on quasimodular forms", J. Ramanujan Math. Soc. 24:3 (2009), 213-233. MR 2011d:11094 Zbl 1206.11052

[Monnier 2002] P. Monnier, "Poisson cohomology in dimension two", Israel J. Math. 129 (2002), 189-207. MR 2003h:53117 Zbl 1077.17018

[Oh 2006] S.-Q. Oh, "Poisson polynomial rings", Comm. Algebra 34:4 (2006), 1265-1277. MR 2007g: 17021 Zbl 1135.17012

[Olver and Sanders 2000] P. J. Olver and J. A. Sanders, "Transvectants, modular forms, and the Heisenberg algebra”, Adv. in Appl. Math. 25:3 (2000), 252-283. MR 2001j:11016 Zbl 1041.11026 
[Pevzner 2008] M. Pevzner, "Rankin-Cohen brackets and associativity”, Lett. Math. Phys. 85:2-3 (2008), 195-202. MR 2010i:53176 Zbl 1167.53075

[Pevzner 2012] M. Pevzner, "Rankin-Cohen brackets and representations of conformal Lie groups", Ann. Math. Blaise Pascal 19:2 (2012), 455-484. MR 3025141 Zbl 1283.11072

[Pichereau 2006a] A. Pichereau, (Co)homologie de Poisson et singularités isolées en petites dimensions, avec une application en théorie des déformations, thesis, Université de Poitiers, Poitiers, 2006, Available at http://dossier.univ-st-etienne.fr/pa11405h/www/PagePersoAP/These_files/fichier.pdf.

[Pichereau 2006b] A. Pichereau, "Poisson (co)homology and isolated singularities", J. Algebra 299:2 (2006), 747-777. MR 2007k:17026 Zbl 1113.17009

[Rochberg et al. 2011] R. Rochberg, X. Tang, and Y.-J. Yao, "A survey on Rankin-Cohen deformations", pp. 133-151 in Perspectives on noncommutative geometry (Toronto, ON, 2008), edited by M. Khalkhali and G. Yu, Fields Inst. Commun. 61, Amer. Math. Soc., Providence, RI, 2011. MR 2838685 Zbl 05994726

[Roger and Vanhaecke 2002] C. Roger and P. Vanhaecke, "Poisson cohomology of the affine plane", J. Algebra 251:1 (2002), 448-460. MR 2003g:17031 Zbl 0998.17023

[Stein et al. 2013] W. A. S. Stein et al., "Sage mathematics software", The Sage Development Team, 2013, Available at http://www.sagemath.org. Version 5.6.

[Unterberger and Unterberger 1996] A. Unterberger and J. Unterberger, "Algebras of symbols and modular forms", J. Anal. Math. 68 (1996), 121-143. MR 97i:11044 Zbl 0857.43015

[Yao 2007] Y.-J. Yao, Autour des déformations de Rankin-Cohen, thesis, École Polytechnique, Centre de Mathématiques Laurent Schwartz, Paris, 2007, Available at http://pastel.archives-ouvertes.fr/ pastel-00002414.

[Zagier 1994] D. Zagier, "Modular forms and differential operators", Proc. Indian Acad. Sci. Math. Sci. 104:1 (1994), 57-75. MR 95d:11048 Zbl 0806.11022

[Zagier 2008] D. Zagier, "Elliptic modular forms and their applications", pp. 1-103 in The 1-2-3 of modular forms, edited by K. Ranestad, Springer, Berlin, 2008. MR 2010b:11047 Zbl 1259.11042

Communicated by Yuri Manin

Received 2013-07-26 Revised 2014-01-20 Accepted 2014-03-24

francois.dumas@math.univ-bpclermont.fr

Clermont Université, Université Blaise Pascal, Laboratoire de mathématiques, BP 10448, F-63000 Clermont-Ferrand, France

Current address: Université Blaise Pascal, Laboratoire de mathématiques, Les Cézeaux, BP 80026, F-63171 Aubière, France

emmanuel.royer@math.univ-bpclermont.fr

Clermont Université, Université Blaise Pascal, Laboratoire de mathématiques, BP 10448, F-63000 Clermont-Ferrand, France

Current address: $\quad$ Université Blaise Pascal, Laboratoire de mathématiques, Les Cézeaux, BP 80026, F-63171 Aubière, France 


\section{Algebra \& Number Theory}

msp.org/ant

\section{EDITORS}

MANAGING EDITOR

Bjorn Poonen

Massachusetts Institute of Technology

Cambridge, USA

\author{
EDITORIAL BOARD CHAIR \\ David Eisenbud \\ University of California \\ Berkeley, USA
}

BOARD OF EDITORS

Georgia Benkart

Dave Benson

Richard E. Borcherds

John H. Coates

J-L. Colliot-Thélène

Brian D. Conrad

Hélène Esnault

Hubert Flenner

Edward Frenkel

Andrew Granville

Joseph Gubeladze

Roger Heath-Brown

Craig Huneke

Yujiro Kawamata

János Kollár

Yuri Manin

Barry Mazur

Philippe Michel

Susan Montgomery
University of Wisconsin, Madison, USA

University of Aberdeen, Scotland

University of California, Berkeley, USA

University of Cambridge, UK

CNRS, Université Paris-Sud, France

University of Michigan, USA

Freie Universität Berlin, Germany

Ruhr-Universität, Germany

University of California, Berkeley, USA

Université de Montréal, Canada

San Francisco State University, USA

Oxford University, UK

University of Virginia, USA

University of Tokyo, Japan

Princeton University, USA

Northwestern University, USA

Harvard University, USA

École Polytechnique Fédérale de Lausanne

University of Southern California, USA
Shigefumi Mori

Raman Parimala

Jonathan Pila

Anand Pillay

Victor Reiner

Peter Sarnak

Joseph H. Silverman

Michael Singer

Vasudevan Srinivas

J. Toby Stafford

Bernd Sturmfels

Richard Taylor

Ravi Vakil

Michel van den Bergh

Marie-France Vignéras

Kei-Ichi Watanabe

Efim Zelmanov

Shou-Wu Zhang
RIMS, Kyoto University, Japan

Emory University, USA

University of Oxford, UK

University of Notre Dame, USA

University of Minnesota, USA

Princeton University, USA

Brown University, USA

North Carolina State University, USA

Tata Inst. of Fund. Research, India

University of Michigan, USA

University of California, Berkeley, USA

Harvard University, USA

Stanford University, USA

Hasselt University, Belgium

Université Paris VII, France

Nihon University, Japan

University of California, San Diego, USA

Princeton University, USA

\section{PRODUCTION}

production@msp.org

Silvio Levy, Scientific Editor

See inside back cover or msp.org/ant for submission instructions.

The subscription price for 2014 is US $\$ 225 /$ year for the electronic version, and $\$ 400 /$ year ( $\$ 55$, if shipping outside the US) for print and electronic. Subscriptions, requests for back issues and changes of subscribers address should be sent to MSP.

Algebra \& Number Theory (ISSN 1944-7833 electronic, 1937-0652 printed) at Mathematical Sciences Publishers, 798 Evans Hall \#3840, c/o University of California, Berkeley, CA 94720-3840 is published continuously online. Periodical rate postage paid at Berkeley, CA 94704, and additional mailing offices.

ANT peer review and production are managed by EditFLOw ${ }^{\circledR}$ from Mathematical Sciences Publishers.

\section{PUBLISHED BY}

mathematical sciences publishers

nonprofit scientific publishing

http://msp.org/

(C) 2014 Mathematical Sciences Publishers 


\section{Algebra \& Number Theory}

Volume $8 \quad$ No. $5 \quad 2014$

Polarization estimates for abelian varieties

DAVID MASSER and GISBERT WÜSTHOLZ

Compatibility between Satake and Bernstein isomorphisms in characteristic $p$

1071

RACHEL OLLIVIER

The final $\log$ canonical model of $\bar{M}_{6}$

1113

FABIAN MÜLLER

Poisson structures and star products on quasimodular forms

1127

FRANÇOIS DUMAS and EMMANUEL ROYER

Affinity of Cherednik algebras on projective space

GWYN BELLAMY and MAURIZIO MARTINO

ALEXANDRU CHIRVASITU

Tetrahedral elliptic curves and the local-global principle for isogenies

1201

BARINDER S. BANWAIT and JOHN E. CREMONA

Local cohomology with support in generic determinantal ideals

1231

Claudiu RAICU and JERZY WEYMAN

Affine congruences and rational points on a certain cubic surface

1259

PIERRE LE BoudeC 